\title{
Glyconanoparticle-DNA Interactions: An Atomic Force Microscopy Study
}

\author{
Peter Eaton, Andrea Ragusa, Caroline Clavel, Cristina T. Rojas, Paul Graham, Raúl V. Durán, and \\ Soledad Penadés*
}

\begin{abstract}
Glyconanoparticles which present carbohydrate and amino groups motifs at their surface were produced. These particles were highly stable and soluble in aqueous solutions. The presence of the carbohydrate groups also allowed the inclusion of more strongly binding groups, without affecting solubility. The binding of a model DNA, plasmid by these nanoparticles was studied by atomic force microscopy, transmission electron microscopy, and gel electrophoresis. Significant differences between the nanoparticles based on their affinities for the DNA were found, with implications for their potential use as nonviral gene delivery agents.
\end{abstract}

Index Terms-Atomic force microscopy, DNA, glyconanoparticles, nucleic acid binders, transmission electron microscopy.

\section{INTRODUCTION}

$\mathbf{T}$ HE BINDING of nanoparticles to DNA is currently a topic of great interest in a number of fields, and has been the focus of many multidisciplinary research efforts [1], [2]. In the context of gene delivery, the aim of the interaction with nanoparticles is to create an alternative to viral gene delivery systems, which face a number of serious problems, such as antigenicity (that is, they commonly evoke a strong immune response) or infectiousness, and safety concerns [3], [4]. On the other hand, nonviral gene delivery agents are often less efficient in transcription [5]-[7]. Such nonviral gene delivery agents aim to condense

Manuscript received April 2, 2007; revised August 11, 2007. This work was supported in part by the EU under CARBONA and GlycoGold Marie-Curie grants and in part by the Spanish Ministry of Education and Science under Grants NAN2004-09125 and CTQ2005-07993. Asterisk indicates corresponding author.

P. Eaton was with Laboratory of Glyconanotechnology, IIQ-CSIC, 41092 Seville, Spain. He is now with REQUIMTE/Departamento de Química, Faculdade de Ciéncias, Universidade do Porto, 4169-007 Porto, Portugal (e-mail: peter.eaton@fc.up.pt).

A. Ragusa, was with Laboratory of Glyconanotechnology, IIQ-CSIC, Americo Vespucio 49, 41092 Seville, Spain. He is now with the National Nanotechnology Laboratory, CNR-INFM, Distretto Tecnologico ISUFI, Via Arnesano, 73100 Lecce, Italy.

C. Clavel was with Laboratory of Glyconanotechnology, IIQ-CSIC, 41092 Seville, Spain and is now at Laboratoire de Chimie Biomoléculaire ENSCM-UMR 5032-cc453, Montpellier, France

C. T. Rojas is with the Instituto de Ciencia de Materiales de Sevilla, CSIC-US, 41092 Seville, Spain.

P. Graham was with Laboratory of Glyconanotechnology, IIQ-CSIC, 41092 Seville, Spain.

R. V. Durán was with Instituto de Bioquímica Vegetal y Fotosíntesis, CSIC-US, 41092 Seville, Spain.

*S. Penadés was with Laboratory of Glyconanotechnology, IIQ-CSIC, 41092 Seville, Spain. She is now with CICbiomaGUNE, Parque Tecnológico, Paseo de Miramon 182, 20009 San Sebastín, Spain (e-mail: spenades@cicbiomagune. es).

Digital Object Identifier 10.1109/TNB.2007.908998 the DNA so that it may more easily enter the cell, while also enabling release of the DNA within the cell.

The interactions between carbohydrates and nucleic acids are of interest because of their role in biological events and the widespread presence of oligosaccharides in antibiotic and anticancer drugs. However, studies on the role of sugars in this interaction are rather scarce [8]. The use of carbohydrate-bearing vectors in transfection has been, however, broadly illustrated [9]-[16]. Galactosylated polyethylenimine [9], [11] and chitosan [10] have been prepared for gene delivery to hepatocytes. Nanosized glycoclusters [12], polyglycoamidoamines [14], [15], and sugar dendritic hybrid polymers [13], [16] as gene delivery vectors were recently investigated. It was found that the nature of the carbohydrate motif had a significant effect on the glycocluster-DNA complex structure. It was also shown that such transfection was highly dependent of the size of the glycocluster-DNA aggregates [12].

Gold nanoparticles represent an ideal scaffold for the creation of DNA-binding systems. Cationic gold nanoparticles that present ammonium groups at the surface were able to bind DNA and prevent RNA transcription by RNA polymerase in vitro [17]. Similarly, polyimine-based gene transfection had been found to be enhanced when the polyimine was conjugated to gold nanoparticles [18]. The authors concluded that this was a result of greater effective molecular weight of the complexes. PEG-modified cationic gold nanoparticles have been used in combination with electroporation for in vivo gene delivery [19]. Most of the nonviral delivery systems are based on the strong electrostatic interactions between the positively charged amino groups and the negatively charged DNA. However, additional molecules able to interact with DNA can modulate this interaction. For example, light-regulated release of DNA has been achieved by means of photolabile gold nanoparticles [20].

In order to elucidate further the characteristics that govern DNA binding by carbohydrates, sugar-functionalized nanoparticles (glyconanoparticles) and amino/sugar hybrid nanoparticles were prepared and their interaction with a linearized plasmid DNA were studied by means of atomic force microscopy (AFM) and transmission electron microscopy (TEM). Glyconanoparticles (GNPs) have been previously prepared and well characterized in our laboratory [21]-[24]. It has been shown that these particles present a multivalent array of carbohydrates at the surface (typically between 60 and 100 molecules), and that they may be used as tools to model carbohydrate-carbohydrate interactions [21], [24].

We report now on the interaction of hybrid glyconanoparticles functionalized with both glucose (Glc) or galactose (Gal) 
monosaccharides and amino ending ethylene glycol chains and a linearized DNA as observed by AFM and TEM microscopy, and gel electrophoresis and the dependence of this interaction on sample preparation.

Preliminary results have shown by TEM and AFM imaging that mixed amino/ $\alpha$-Gal $(1: 1)$ nanoparticles are highly efficient DNA-binders. Furthermore, they are able to condense DNA into a compact globular shape, which is a desired property for gene transfection agents.

\section{EXPERIMENTAL PROCEDURE}

\section{A. Chemicals}

All chemical reagents were used without further purifications. All water used was DNAse and RNAse free $18 \mathrm{M} \Omega$ water (Sigma), which was further sterilized by autoclaving for $30 \mathrm{~min}$ at $121{ }^{\circ} \mathrm{C}$ before use. AFM images were obtained with a Topometrix Explorer atomic force microscope or a Veeco multimode IVa AFM (both from Veeco Metrology, Santa Barbara, CA), using silicon cantilevers (Mikromasch, Tallinn, Estonia) with a resonance frequency around $210 \mathrm{kHz}$ using the computer controlled noncontact mode. No significant differences were noticed between images from the two machines. In order to image isolated DNA molecules, it was necessary to use very dilute solutions $(300 \mathrm{pM})$ due to the large size of the molecules. AFM images of the nanoparticles were obtained by drying a $3 \mu \mathrm{L}$ droplet of a $10 \mathrm{nM}$ to $10 \mu \mathrm{M}$ solution deposited on the mica. TEM imaging was carried out with a Philips CM200 microscope working at $200 \mathrm{kV}$ with a LaB6 filament. Samples were deposited on standard carbon coated copper grids and allowed to air-dry. Particle size was measured using an automatic image analyzer. ${ }^{1}$

\section{B. Preparation of the Molecules and Gold Glyconanoparticles}

The 17-amino-1-thio-hexa(ethylene glycol) was synthesized starting from hexa(ethylene glycol) in a five-step synthesis (see supplementary information). All thiol-conjugated saccharides (neoglycoconjugates) were synthesized by conventional glycosydation methods and isolated as the disulphide derivative (see supplementary information). The corresponding glyconanoparticles (GNPs) were prepared as described previously [22]. The nanoparticles as prepared were characterized by AFM, TEM, and ${ }^{1} \mathrm{H}-\mathrm{NMR}$ (see supplementary information).

\section{Preparation of the Linearized Plasmid}

pACC plasmid, a $5.1 \mathrm{~kb}$ pBluescript SK (+) (Stratagene) derivative was digested with Nsi1 restriction endonuclease (Roche) to prepare the linearized DNA fragment and purified using standard procedures. Particular care was taken to remove proteins from the DNA sample, so as to avoid mistaking such debris for nanoparticles in the later analysis. All DNA solutions were $300 \mathrm{pM}$ in concentration of DNA as estimated by 260-280 $\mathrm{nm}$ absorption spectrophotometry [25].

\section{Preparation of Glyconanoparticle-DNA Complexes}

Method 1. A $300 \mathrm{pM}$ solution of DNA in $1 \mathrm{mM}$ tris- $\mathrm{HCl}$ buffer at $\mathrm{pH} 7.4$ and a $4 \mu \mathrm{M}$ water solution of glyconanoparti-

${ }^{1}$ [Online]. Available: http://rsb.info.nih.gov/ij/ cles were mixed and allowed to interact for $10 \mathrm{~min}$ at room temperature. The solutions were mixed in 1 to 1 volume ratios, and the total volume was 3-6 $\mu \mathrm{L}$ for AFM analysis and $10-20 \mu \mathrm{L}$ for TEM analysis. For the AFM experiments, the buffer was $1 \mathrm{mM}$ tris- $\mathrm{HCl}$ buffer, $\mathrm{pH} 7.4,10 \mathrm{mM} \mathrm{NiCl} 2$, as $\mathrm{Ni}^{2+}$ is needed to aid binding of DNA onto mica. A droplet of 3-6 $\mu \mathrm{L}$ of the solution was deposited onto freshly cleaved mica and allowed to stand for 2 min to fix the DNA to the surface. The sample was then further rinsed with water $\left(5 \mathrm{~mL} \mathrm{H}_{2} \mathrm{O}\right)$ to remove salts and then was dried with a stream of argon and further dried under vacuum before imaging. Samples for TEM analysis were simply deposited from the mixed solution onto standard carbon coated copper grids and allowed to air-dry.

Method 2. 3-6 $\mu \mathrm{L}$ from a $300 \mathrm{pM}$ solution of DNA in $1 \mathrm{mM}$ tris- $\mathrm{HCl}$ buffer, $\mathrm{pH} 7.4$, with $10 \mathrm{mM} \mathrm{NiCl} 2$ was deposited onto freshly cleaved mica. The sample was allowed to stand for $2 \mathrm{~min}$, then washed copiously with water $\left(5 \mathrm{~mL} \mathrm{H}_{2} \mathrm{O}\right)$, dried with a stream of argon, and further dried under vacuum before imaging. An aqueous solution of nanoparticles 3-6 $\mu \mathrm{L}$ of a $4 \mu \mathrm{M}$ solution was then added and allowed to interact with the fixed DNA for 10 min followed by drying by argon and vacuum before imaging.

\section{E. Gel Electrophoresis}

GNP stock solutions at $1 \mathrm{mg} / \mathrm{mL}$ were diluted to the desired concentration and $10 \mu \mathrm{L}$ of each solution mixed with $10 \mu \mathrm{L}$ $(20 \mathrm{ng}$ ) of pDNA. After resting for $15 \mathrm{~min}$, the samples were loaded into the wells of a $0.6 \%$ agarose gel and the electrophoresis performed in TAE buffer $(90 \mathrm{mM}$ Tris-acetate and $2 \mathrm{mM}$ EDTA, $\mathrm{pH}$ 8.0) at $125 \mathrm{~V}$ for $30 \mathrm{~min}$. The gel was stained with ethidium bromide and illuminated with a UV illuminator to observe the pDNA. Linearized pACC-Nsi1 plasmids consist of approximately 5100 base pairs, as confirmed by the corresponding line in the DNA molecular weight ladder.

\section{RESUlTS AND DISCUSSION}

We used the monosaccharides glucose (Glc) and galactose (Gal) as the sugar motifs and the 17-amino-1-thio-hexa(ethylene glycol) as the amino-bearing chain (Scheme 1). Synthesis of the 17-amino-1-thio-hexa(ethylene glycol) was accomplished starting from hexa(ethylene glycol) by modification of previously reported reaction sequences [22].

The sugars were transformed into both $\alpha$ - and $\beta-1-\mathrm{O}-(2-$ thioethyl) glycosides to see the influence of the sugar anomeric configuration in the interaction. The neoglycoconjugates 1-O-(2-thioethyl) of $\alpha$ - and $\beta$-galactose $(\alpha$-GalC2S and $\beta$-GalC2S), $\alpha$ - and $\beta$-glucose ( $\alpha$-GlcC2S and $\beta$-GlcC2S $)$ were prepared by different glycosydation methods. Synthesis of the 1-O-(2-thio-ethyl)- $\alpha$-D-galactose ( $\alpha$-GalC2S) involved initially a Fischer glycosylation of $\mathrm{D}(+)$-galactose with bromoethanol. The resulting product was then acetylated using acetic anhydride. Nucleophilic displacement of the bromo group was then carried out using potassium thioacetate. The required product was obtained by deacetylation using sodium methoxide in methanol. Synthesis of the 1-O-(2'-thio-ethyl)$\alpha$-D-glucose ( $\alpha$-GlcC2S) was carried out using the same procedure, but starting from $\mathrm{D}-(+)$-glucose. A similar route was used to obtain the 1-O-(2'-thio-ethyl)- $\beta$-D-glucose $(\beta$-GlcC2S $)$ and 

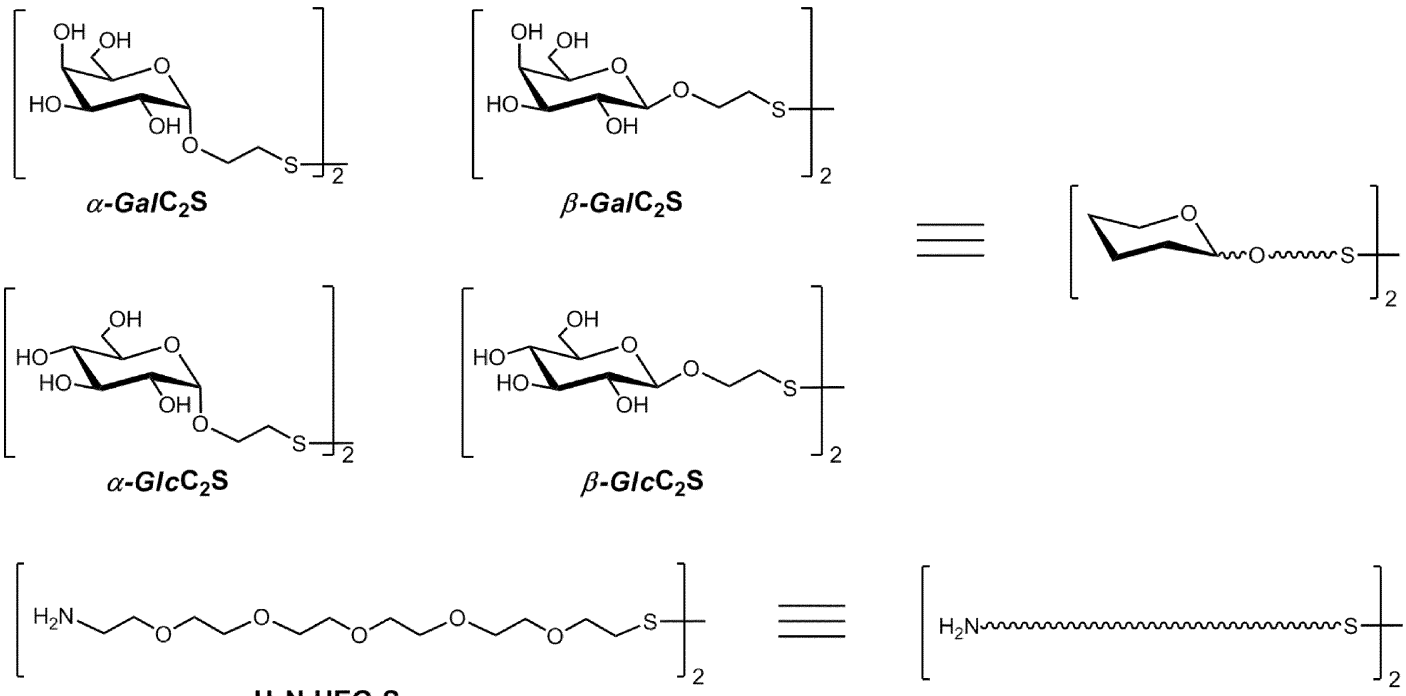

$\mathrm{H}_{2}$ N-HEG-S
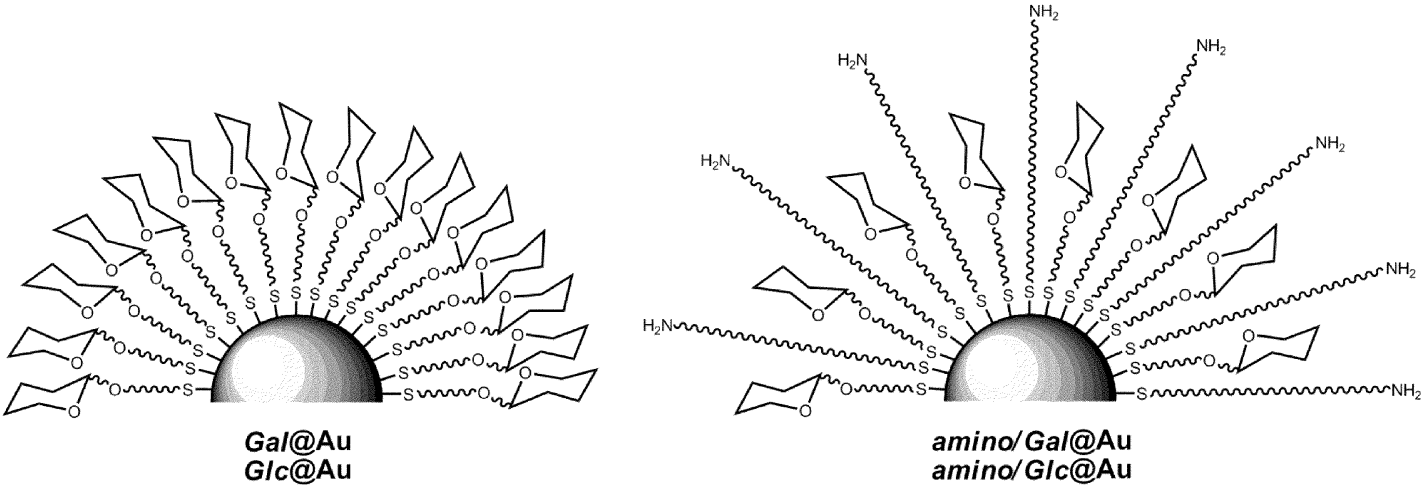

Scheme 1. Chemical structures of the $\alpha$ - and $\beta$-Gal and $\alpha$ - and $\beta$-Glc conjugates, of the amino-hexa(ethylene glycol) chain, and schematic representation of the pure sugar and mixed amino/sugar glyconanoparticles.

the 1-O-(2'-thio-ethyl)- $\beta$-D-galactose ( $\beta$-GalC2S) derivatives but the commercially available peracetylated $\beta$-D-glucose and $\beta$-D-galactose compounds were used as starting material. The participation of the acetyl protecting group in position 2 contributed, through the formation of an intramolecular oxazolidine ring, to the formation of the $\beta$ anomer. Again, the brominated derivatives were treated with potassium thioacetate and subsequent removal of the acetyl protecting groups with sodium methoxide yielded the corresponding disulfides (see supplementary material).

Gold glyconanoparticles (GNPs) were prepared as previously described [22]. GNPs of glucose and galactose were prepared using the corresponding $\alpha$ - and $\beta$ - neoglycoconjugates. Nanoparticles prepared only with the 1-thio-17-amino hexa(ethylene glycol) chain were extremely unstable in solution, precipitating over a broad $\mathrm{pH}$ range, in contrast to the stability of 2-aminoethanothiol protected gold nanoparticles previously reported [26]. Pure sugar GNPs capped with $\beta$-GlcC2S, $\alpha$-GalC2S and $\beta$-GalC2S were also prepared. Attempts to prepare the $\alpha$-GlcC2S GNPs were unsuccessful. The hybrid amino/Gal and amino/Glc nanoparticles were prepared either starting from a methanolic solution of $1: 1$ equivalents of neoglycoconjugate/amino chain (as for the amino/ $\alpha$-Gal GNPs) or by ligand exchange of the pure saccharidic GNPs with the thiolated amino chain (as for the amino/ $\beta$-Gal and the amino/ $\beta$-Glc GNPs), resulting in an approximate density of the amino functionalized chain on the surface of $50 \%$. Analysis of the ${ }^{1} \mathrm{H}-\mathrm{NMR}$ spectrum of the resulting nanoparticles as well as of the mother liquor confirmed that the particles contained a 1:1 ratio of sugar/amino linker. The neoglycoconjugates confer high solubility to the particle, avoiding the precipitation problems found with pure amino nanoparticles. TEM images show that the gold clusters have mean diameters of $1.8 \mathrm{~nm}$ ( $\alpha$-Gal), $2.7 \mathrm{~nm}(\beta$-Gal), 2.2 ( $\beta$-Glc), $1.1(\mathrm{amino} / \alpha-\mathrm{Gal}), 2.7$ $(\mathrm{amino} / \beta$-Gal), and $2.1 \mathrm{~nm}(\mathrm{amino} / \beta$-Glc) (see supplementary material). According to the dimension of the metallic core this would represent an estimated total number of ligands ranging from about 51 for the smallest GNPs to about 335 for the biggest ones. [27]

The DNA fragment was chosen based on its convenient size for AFM experiments. The $5.1 \mathrm{~kb}$ linearized pACC DNA fragment would theoretically have a contour length of $1.7 \mu \mathrm{m}$. AFM measurements tend to exaggerate the length of such molecules slightly [28]. However, the AFM scanner used had a maximum scan range of $2.3 \mu \mathrm{m}$, so could easily image a whole DNA molecule.

Using the deposition and imaging conditions detailed in the experimental section, high-quality images of DNA and glyco- 

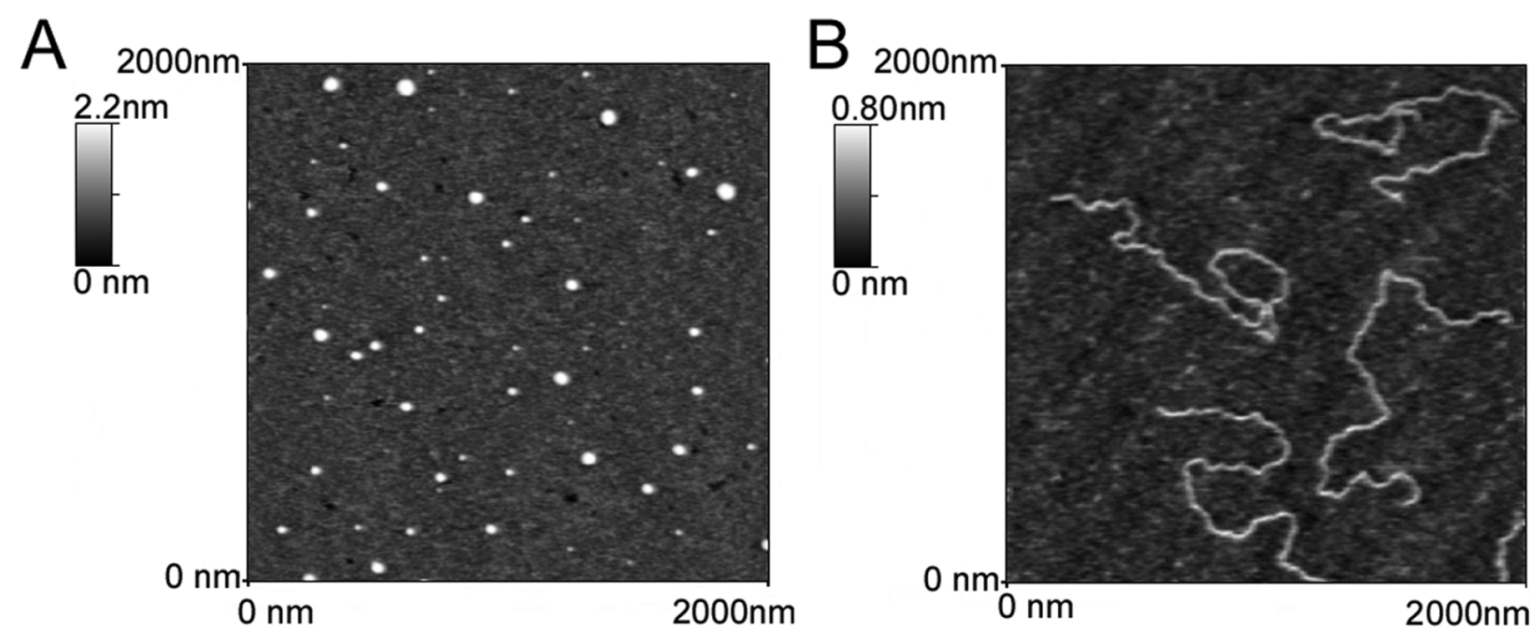

Fig. 1. AFM image of: (A) linearized pACC DNA fragments used in this work deposited from $300 \mathrm{pM}$ solution and (B) $\beta$-Glc GNPs deposited from $60 \mathrm{nM}$ solution.

nanoparticles could be obtained. Fig. 1(A) and (B) shows typical images of the plasmid DNA fragments and of the $\beta$-Glc GNPs, respectively. It can be seen that the procedures for deposition resulted in clean images. All glyconanoparticles measured had heights between 0.5 and $5 \mathrm{~nm}$. The average particle height was $2.47 \mathrm{~nm}(\beta$-Glc), $0.95 \mathrm{~nm}(\alpha-\mathrm{Gal}), 2.1 \mathrm{~nm}(\beta$-Gal), $1.96 \mathrm{~nm}(\mathrm{amino} / \alpha-\mathrm{Gal}), 3.3 \mathrm{~nm}(\mathrm{amino} / \beta-\mathrm{Gal})$. Particle width as measured by AFM is largely a function of the radius of the AFM tip used, due to convolution of tip shape with sample morphology. According to previous results, all the particles are approximately spherical, so real width and height should be identical [24].

Interaction studies were performed in two ways. Plasmid and glyconanoparticles were mixed in solution and allowed to interact before deposition onto the surfaces (Method 1). Alternatively, the plasmid was deposited onto the mica surface and the first image recorded. Then glyconanoparticles were added from aqueous solution and allowed to interact with the fixed DNA for $10 \mathrm{~min}$ (Method 2). The first method employed allows the nanoparticles and DNA to interact in solution before depositing onto mica for imaging. Method 1 is more relevant to the interactions in, e.g., a test tube-based biosensor or gene delivery application, while the advantage of method 2 was that the fixation of the DNA to the surface allowed less ambiguous imaging by AFM.

When DNA and amino/ $\alpha$-Gal GNPs were allowed to interact in solution before depositing onto mica (Method 1), AFM images showed that the concentration of both free DNA and nanoparticles have been drastically reduced [Fig. 2(A)] and some very large features with globular morphology appeared on the surface. No individual DNA or GNPs could be observed. It was therefore suspected that these features represented aggregates formed between the nanoparticles and DNA. However, as this was not clear from the AFM images alone, the solution of amino/ $\alpha$-Gal GNPs-DNA was studied by TEM. Fig. 2(B) shows the corresponding TEM image where it is clear that the globular features contain the nanoparticles [black dots in Fig. 2(B)]; moreover, close inspection shows that all the nanoparticles were contained within the gray halos, which indicate the presence of organic material. We interpret this as indicating that all nanoparticles are associated with the DNA. Comparison of Fig. 2(A) and (B) shows that the same features were seen by both AFM and TEM. Any differences in the images can be accounted for by the different imaging techniques; in both cases globular features of approximately 50 to $200 \mathrm{~nm}$ diameter could be seen. The interaction between the DNA molecules and the nanoparticles was also observed following method 2. In this method DNA was first deposited on the mica before the nanoparticles were added. This allowed the DNA to adopt an extended configuration on the mica surface, which was checked by AFM before addition of nanoparticles [as seen in Fig. 1(A)]. Addition of nanoparticles then resulted in images showing the nanoparticles preferentially adopted positions along the DNA molecule. A typical image of the resulting structures obtained by this method with hybrid amino/ $\alpha$-Gal nanoparticles is shown in Fig. 2(C). In this figure, one may see that while there were some individual, unattached nanoparticles, the majority of them aggregated around the DNA molecules on the surface. It is worth noting that this image shows some "uncoated" DNA (the very thin white lines) as well as the regions of DNA that have many nanoparticles attached. This suggests that initial binding of the hybrid amino/ $\alpha-\mathrm{Gal}$ nanoparticles to DNA causes further nanoparticles to aggregate around the DNA-nanoparticle complexes. The mechanism of this action is similar to that observed in the interaction between DNA and a great excess of lysine-modified gold nanoparticles [29] or in a plasmid deposited on poly-L-ornithine-coated mica after mixing with polyethylenimine [30]. Fig. 2(D) shows a typical image of a sample further treated after initial imaging by washing with water. This process appears to have broken up the initial aggregates leading to more unattached nanoparticles, but also to the formation of large compact globular aggregates, which may be the beginning of the compaction process, i.e., the structures in Fig. 2(D) were intermediate between those in Fig. 2(C) and (A). It seems that if the nanoparticles and DNA were left for longer to interact (and the DNA was not partially fixed to the mica), large globular aggregates such as seen in Fig. 2(A) would be seen, possibly with the eventual binding of 

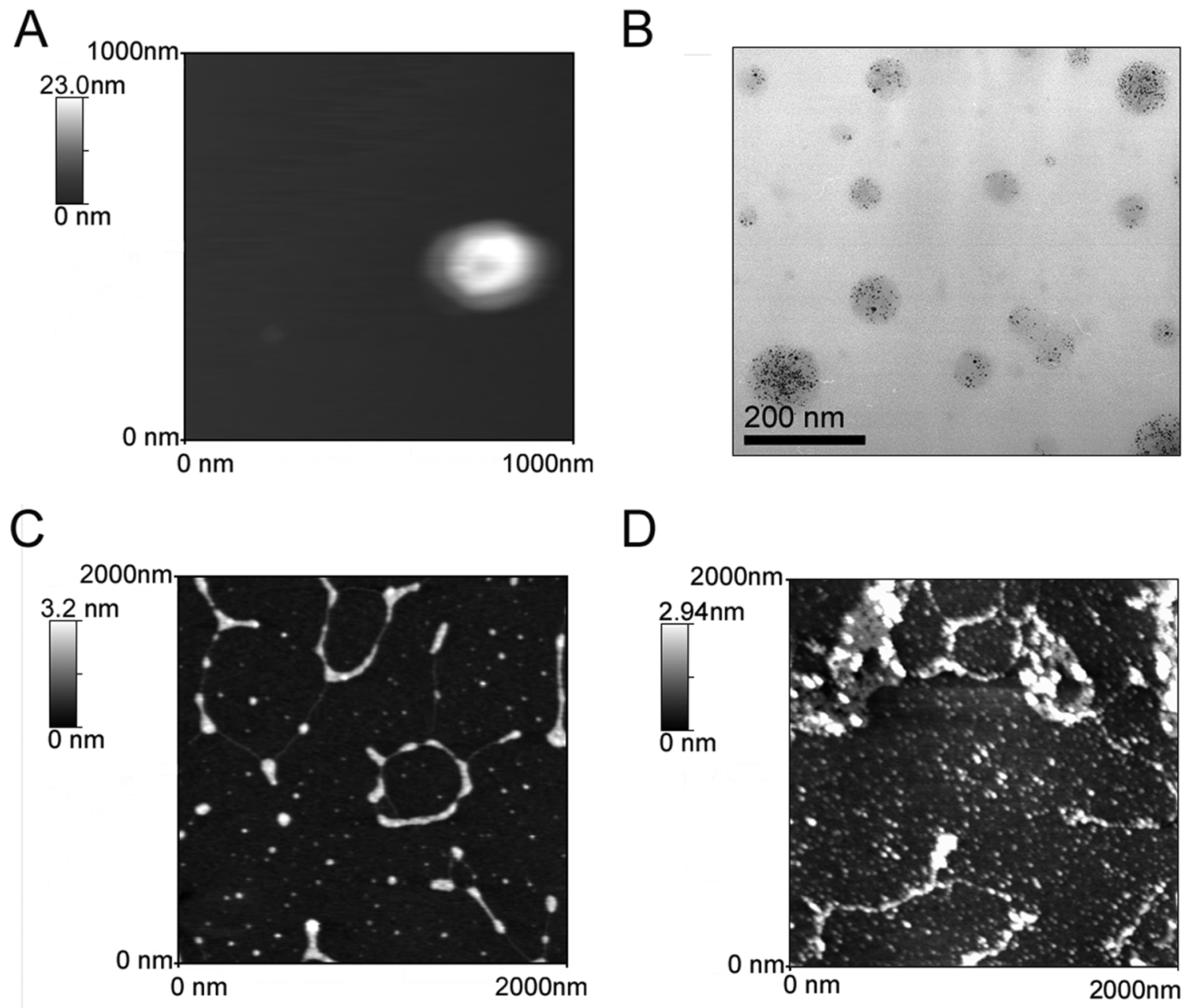

D
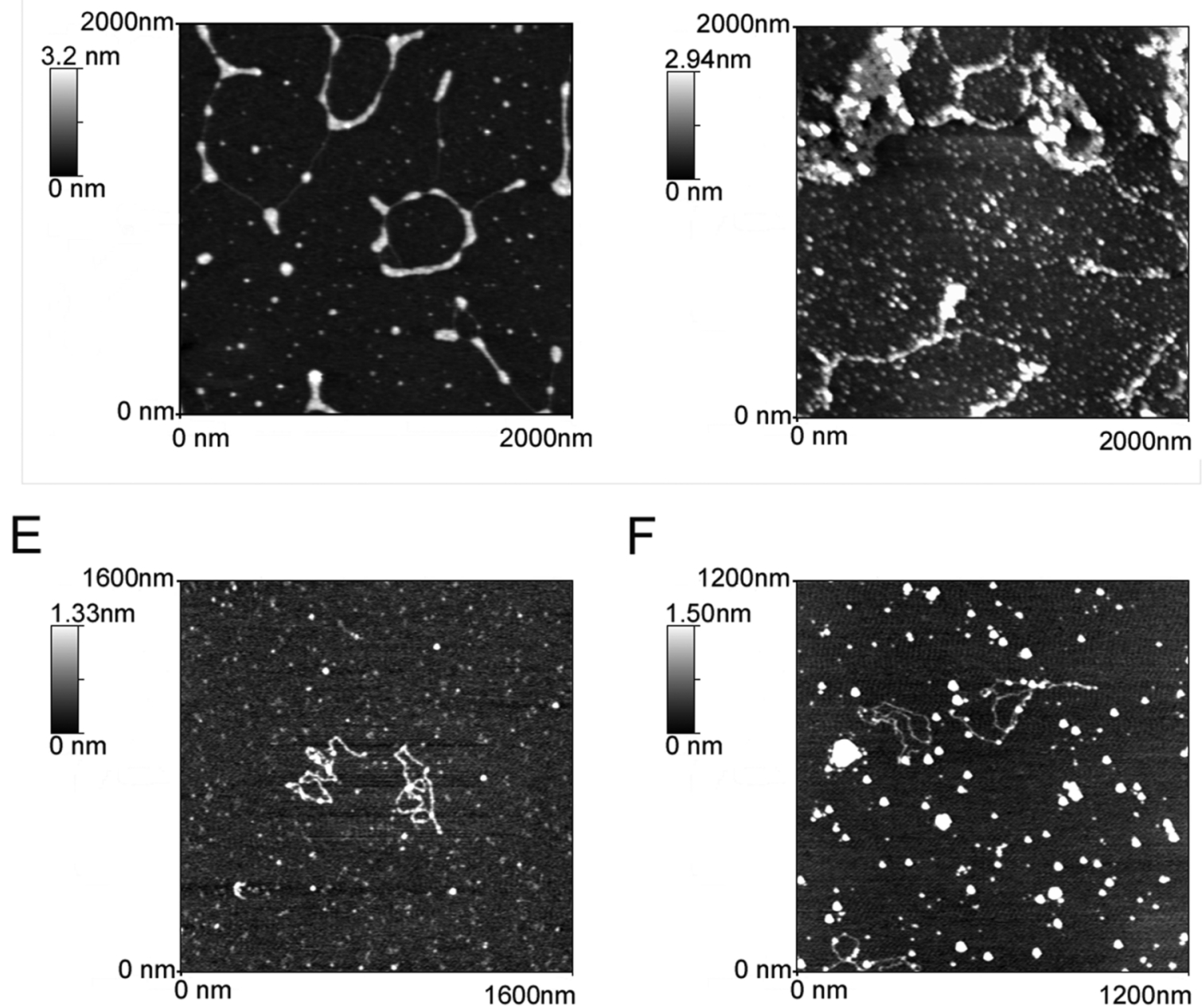

Fig. 2. Hybrid amino/ $\alpha$-Gal glyconanoparticles and DNA deposited from solution (method 1): (A) imaged by AFM and (B) by TEM, and AFM images of hybrid amino/ $\alpha$-Gal nanoparticles deposited onto fixed DNA (method 2) before (C) and after (D) washing with water, and of hybrid amino/ $\beta$-Gal nanoparticles deposited on to DNA fixed on mica before $(\mathrm{E})$ and after $(\mathrm{F})$ washing.

all unattached nanoparticles. In Fig. 2(E) and (F) are shown the images of the $\beta$-Gal nanoparticles on fixed mica, and the same sample after washing, respectively. It may be seen that in comparison with the amino/ $\alpha-\mathrm{Gal}$, the amino/ $\beta$-Gal nanoparticles [Fig. 2(E)] exhibited much less DNA binding. Although particles do appear to have bound the DNA chains, there are many more unbound particles present than in Fig. 2(C). However, after washing several large aggregates appeared [Fig. 2(F)], suggesting that over time compaction of the DNA also occurs for these glyconanoparticles.

The interactions between the pure sugar $\alpha$-Gal, $\beta$-Gal and $\beta$-Glc nanoparticles with DNA were also studied as for the hybrid nanoparticles. Typical results are shown in Fig. 3. Fig. 3(A) and (B) correspond to $\beta$-Glc nanoparticles on trapped 

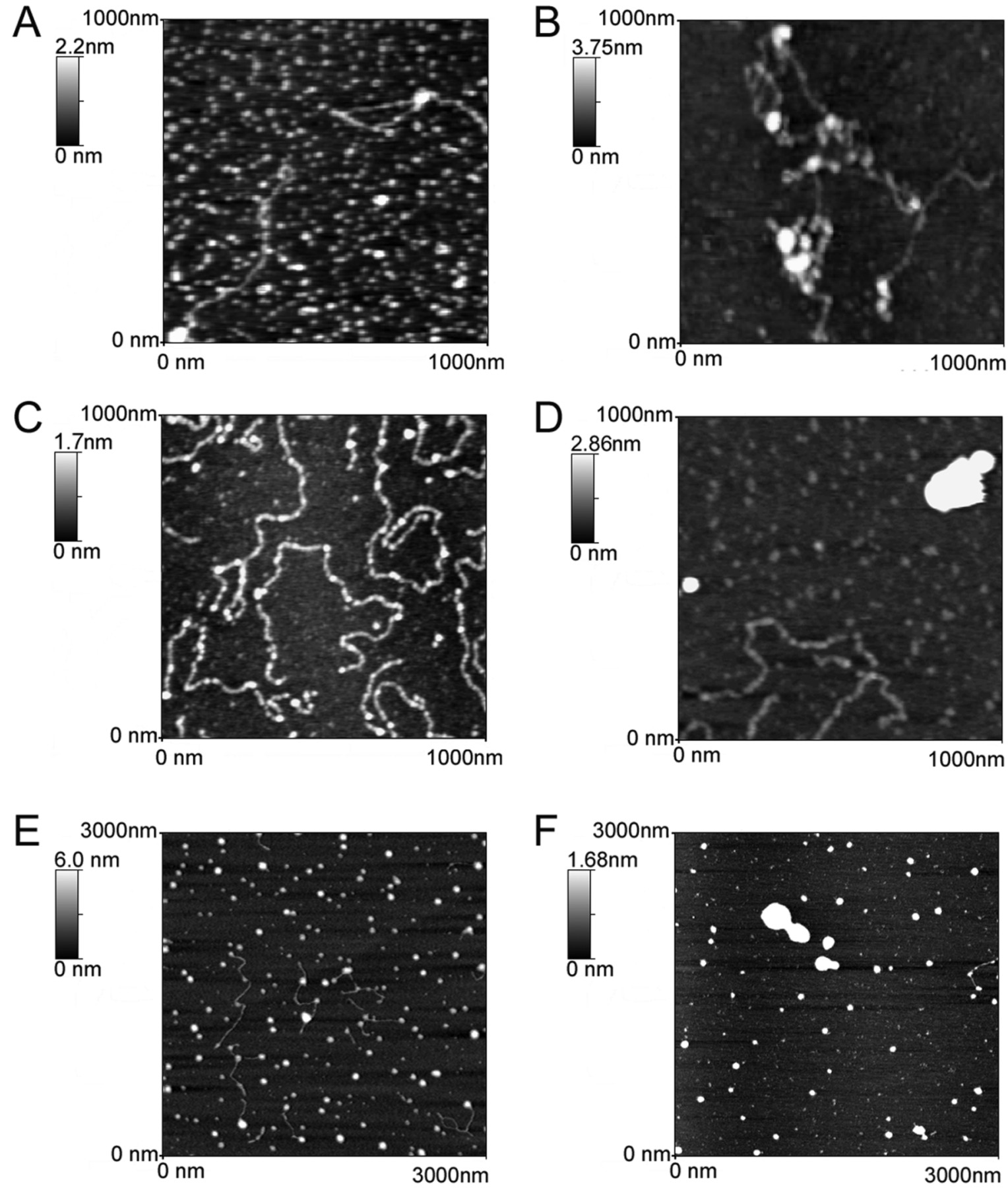

Fig. 3. AFM images of $\beta$-Glc glyconanoparticles deposited onto fixed DNA before (A) and after (B) washing, of $\alpha$-Gal nanoparticles deposited onto fixed DNA on mica before (C), and after (D) washing, and of $\beta$-Gal nanoparticles deposited onto fixed DNA on mica before (E) and after (F) washing.

DNA before washing (Method 2) and on trapped DNA after washing. Fig. 3(C) and (D) correspond to $\alpha$-Gal nanoparticles on trapped DNA before washing and on trapped DNA after washing. Fig. 3(E) and (F) are of $\beta$-Gal nanoparticles on trapped DNA before washing and on trapped DNA after washing. From Fig. 3(A), one may see both many unbound $\beta$-Glc nanoparticles, and some particles lying along the DNA strands; however, selectivity to the DNA in this case seems low. An image of the sample after washing with water is shown in Fig. 3(B). In this image, far fewer nanoparticles lie on the mica, and close examination reveals that nanoparticles are preferentially aligned along the DNA molecules. Furthermore, it may be seen that the DNA-nanoparticle complexes have become quite large (white features in the center of the image), with the DNA strands protruding from these features. It seems likely that these globular features are the forerunners of even larger globular aggregates formed in solution (Fig. 2). Images of a sample of $\beta$-Glc GNPs and DNA deposited from solution (Method 1) displayed also large aggregates, as well as both free nanoparticles and free DNA (see supplementary information). This contrasts with similar images from the hybrid nanoparticles-DNA complex deposited from solution [Fig. 2(A)], which 
showed no free DNA fragments or nanoparticles. Fig. 3(A) and (B) show that the $\beta$-Glc nanoparticles did interact with DNA; but much less strongly than the hybrid nanoparticles.

The image of the interaction of $\alpha$-Gal GNPs with DNA [Fig. 3(C)] shows the nanoparticles aligned along the DNA chains, though again, no formation of aggregates as in the case of the amino/ $\alpha-\mathrm{Gal}$ nanoparticles (Method 1) was observed. However, after washing with water large aggregates can be observed in the image [Fig. 3(D)]. Similarly to the case of $\beta$-Glc nanoparticles, the images obtained from the solution-deposited GNPs-DNA complex, showed both free nanoparticles and DNA in contrast to the results obtained from the hybrid nanoparticles-DNA interaction in solution (see supplementary information).

Fig. 3(E) and (F) show that for the $\beta$-Gal glyconanoparticles, before washing a relatively large number of nanoparticles were not bound to the DNA chains, although each DNA molecule had several nanoparticles bound to it. After washing, however, the percentage of unbound nanoparticles decreased greatly, and large features appeared in the images. This showed that the nanoparticles bound the DNA relatively weakly but formed some larger aggregates after washing. Taken together, the data on the carbohydrate-only nanoparticles indicate that the form of the saccharide present was not important for sugar-only nanoparticles.

Comparison of Figs. 2 and 3 reveals significant differences in the behavior between the nanoparticles that had the amino chain, and those that did not. Although it appears that a higher proportion of the $\alpha$-Gal nanoparticles were bound preferentially to the DNA than the $\beta$-Gal, the difference between these two samples and the hybrid amino $/ \alpha$-Gal nanoparticles is more remarkable. Under the conditions of method 2 and at the same concentration of added GNPs, the carbohydrate-only nanoparticles showed a slight preference for assembling along the DNA chains [Fig. 3(A), (C), and (E)], when compared with the extremely high affinity for the DNA of hybrid nanoparticles [Fig. 2(C)]. This strong difference is not observed as comparing the behavior of the pure sugar $\beta$-Glc, $\alpha$-Gal, and $\beta$-Gal GNPs. However, there were a higher percentage of nanoparticles aligned along the DNA molecules in the images of $\alpha$-Gal nanoparticles compared to the other two glyconanoparticles [see Fig. 3(A), (C), and (E)]. The effect was much more pronounced in the case of the hybrid amino/ $\alpha$-Gal GNPs, however, leading us to conclude that the combination of the $\alpha$-Gal and amino groups gave a synergistic effect. Electrostatic interactions between the amino groups of the GNPs and the phosphate groups along the DNA backbone may be the main driving force for this association. However, sugar configuration seems to also play also a role, as the $\alpha$-Gal nanoparticles were considerably stronger DNA binders than the $\beta$-Gal particles. While it may be postulated that the immobilized DNA does not act like free DNA in solution, it has been shown that DNA which is bound to the surface by divalent cations is, while "fixed" to the surface, able to engage in complex biologically relevant reactions, which also occur in solution, such as DNA cleavage by DNAse [31] and specific protein binding [32]. In this context, it is feasible that similar reactions with the nanoparticles may occur on the surface and in solution.
TABLE I

ANALYSIS OF AFM IMAGES

\begin{tabular}{|l|c|c|}
\hline Sample & $\begin{array}{l}\text { Change in density of } \\
\text { unbound GNPs } \\
\left.\text { GNP per } \mu \mathrm{m}^{2}\right)^{\mathrm{a}}\end{array}$ & $\begin{array}{l}\text { Density on DNA } \\
\text { chain } \\
\mu \mathrm{m})^{\mathrm{b}}\end{array}$ \\
\hline$\alpha$-gal/ $/ \mathrm{NH}_{2}$ & 489 & \multicolumn{2}{|c|}{$\mathrm{NA}^{\mathrm{c}}$} \\
\hline$\beta$-gal/ $\mathrm{NH}_{2}$ & 90 & 15 \\
\hline$\beta$-glc & 11 & 14.7 \\
\hline$\alpha$-gal & 412 & 27.4 \\
\hline$\beta$-gal & 40 & 13.2 \\
\hline
\end{tabular}

a: Number density of unbound nanoparticles in images after washing expressed as a percentage of number before washing. b: Mean number of nanoparticles found along DNA chains per $\mu \mathrm{m}$ before washing. c: Data not available as particles could not be distinguished; see text.

In order to further understand the differences in binding of the glyconanoparticles to DNA, we attempted to image also the nanoparticle/DNA complexes formed in solution (Method 1) by TEM.

In comparison to the compact globular complexes seen in the case of the hybrid amino/ $\alpha$-Gal nanoparticles [Fig. 2(B)], for all the carbohydrate-only nanoparticles, any complexes seen had a less well-defined shape. In general, it was very difficult to obtain images of these complexes. In some cases, the samples were extremely quickly damaged by the electron beam, making imaging impossible, while in others the samples were quite stable to imaging.

As far as we are aware, no differences in sample preparation gave rise to this differing behavior, and so we concluded that the difference may be due to the nature of the DNA/nanoparticle complexes. The "compaction" by the amino-carrying nanoparticles probably protects the DNA from the rapid burning that occurred to samples in the TEM in the case of the pure carbohydrate $\beta$-Glc and $\alpha$-Gal nanoparticles. Finally, although as shown in Fig. 2, in the hybrid amino/ $\alpha$-Gal nanoparticles and DNA solutions all the DNA was bound into large globular particles, in the case of the solutions of $\alpha$-Gal, $\beta$-Glc, amino/ $\beta$-Gal, and amino/ $\beta$-Glc nanoparticles with DNA, AFM analysis revealed the presence of free DNA (and free nanoparticles) when the solution was dried onto mica. These were never seen in the case of the hybrid amino/ $\alpha$-Gal nanoparticles.

In addition to the qualitative observation of the AFM images, it is instructive to measure the change in density of nanoparticles on the samples upon washing, which shows considerable differences between the samples. Table I shows the change in mean density of unbound nanoparticles (i.e., those not touching a strand of DNA) for each sample before, and after washing.

The results show that upon washing the number of free nanoparticles is greatly reduced for both the $\beta$-monosaccharide functionalized nanoparticles, reducing the density of GNPs to $11 \%$, and $40 \%$ of their initial values for $\beta$-gluco and $\beta$-galacto particles, respectivelyIn both these cases, large aggregates started to appear on the samples, after washing [see Fig. 3(B) and (F)]. We may interpret this as indicating either than the particles were simply washed away, or that the "free" particles become bound up in these large clusters. In the case of both the mixed amino/alpha galactose and alpha galactose particles, 
there was actually an increase in free particle count upon washing. These two samples were those that in the initial images [Figs. 2(C) and 3(C)], showed the highest number of nanoparticles bound to the DNA. Therefore, what happens upon washing is that the binding of some of the nanoparticles to the DNA is broken up, and at least some of those "released" nanoparticles remain on the mica surface. This may be seen as surprising, as after $10 \mathrm{~min}$ in solution, the $\alpha$-galacto/amino GNPs had formed very large clusters [see Fig. 2(A) and (B)]. This may only mean, however, that the condensing into large cluster does not occur easily with the DNA trapped on the surface in this case.

Finally, the $\beta$-galacto/amino nanoparticles showed intermediate behavior, as the number of free nanoparticles before and after washing was very similar (only $10 \%$ loss, which may be attributed to "washing away"). This suggests than upon a short treatment time, these nanoparticles neither came unbound from the DNA and neither did they start to form large clusters.

Counting of the number of nanoparticles bound to each DNA strand imaged (before washing) gave results that in general varied little from one type of nanoparticle to another, most particles giving results around $15 \pm 3$ nanoparticles bound per $\mu \mathrm{m}$ of exposed DNA. This density of binding is rather low, considering the size of each nanoparticle, but is perhaps reduced due to the trapping of the DNA on the mica surface, reducing the number of available binding sites. The exception to this was the alpha galacto nanoparticles which bound almost double the number of nanoparticles $(27 \pm 14)$ per $\mu \mathrm{m})$. The amino/ $\alpha$-galacto NPs of course showed a completely different type of binding with the nanoparticles clustering together very tightly such that the AFM is unable to distinguish them [Fig. 2(C)]. It was not possible to asses accurately the number of nanoparticles in these clusters around the DNA chains, but comparision of the volume of the clusters with the volume of chains with bound $\alpha$-galacto GNPs suggests there are many more nanoparticles bound in the case of the mixed nanoparticles.

The influence of the carbohydrate and of the amine-chains in complexing pACC DNA was also evaluated by gel shift electrophoresis in $0.6 \%$ agarose gels using various weight/weight (w/w) ratios of GNP to pDNA. Each GNP was combined with the DNA at w/w ratios $0,2.5,5,10,20,40,50$, and 100 for $15 \mathrm{~min}$ before being loaded into the gel and electrophoresed. The GNPs without amine-ending chain were used as negative control in all experiments since they were not expected to be able to charge-neutralize, hence retain, the DNA. On the other hand all the amino/sugar GNPs were able to inhibit the electrophoretic mobility of the plasmid upon addition of the cationic GNPs, although at different concentrations. However, the degree of retention strongly depended on the type of GNPs.

The ability of the GNPs to completely inhibit the DNA from progressing toward the positive electrode indicates either that the complexes formed were completely charge-neutralized or that the resultant complexes were too large to enter the gel. However, in some cases the formation of intermediate assemblies, due either to partial charge-neutralization or to the formation intermediate complexes with different molecular weights, was noticed. Fig. 4(A), (C), and (E) reveal that, as expected, none of
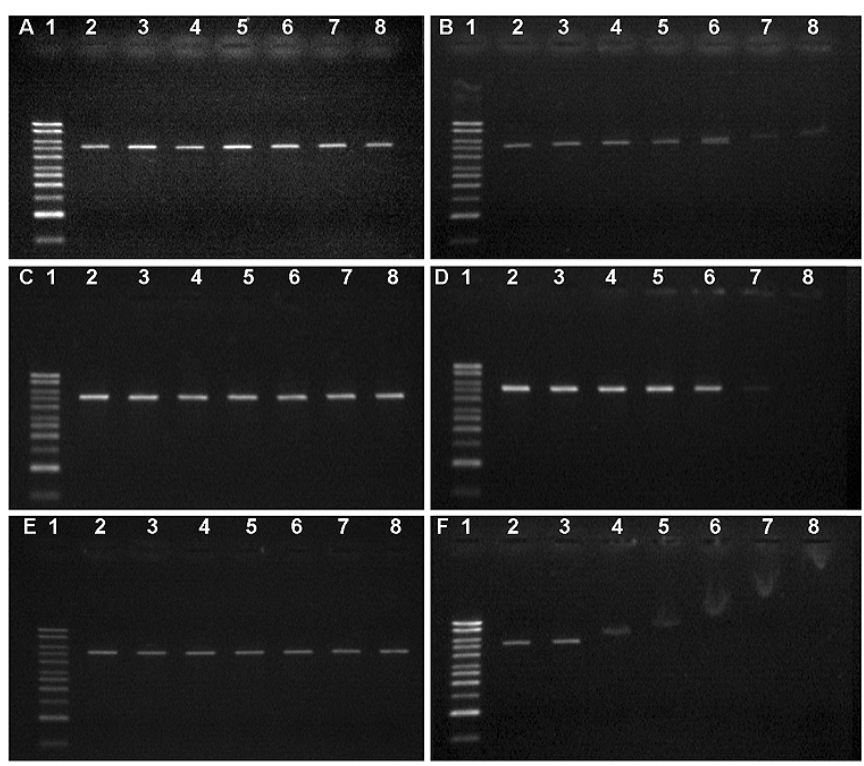

Fig. 4. Agarose gel electrophoresis of GNPs-pDNA complexes. Line assignments are as follows: (A) control experiments with $\alpha$-Gal GNPs; (B) experiments with mixed amino/ $\alpha$-Gal GNPs; (C) control experiments with $\beta$-Gal GNPs; (D) experiments with mixed amino/ $\beta$-Gal GNPs; (E) control experiments with $\beta$-Glc GNPs; (F) experiments with mixed amino/ $\beta$-Glc NPs. Column assignments are as follows: line 1) represents the DNA ladder; lines 2) to 8) represent GNPs-pACC-Nsi1 complexes at w/w ratios of 0, 2.5, 10, 20, 40, 50 , and 100 , respectively.

the GNPs functionalized with only carbohydrates is able to bind and neutralize pDNA at any concentration used. Fig. 4(B), (D), and $(F)$ refer to the binding ability of the mixed amino/sugar nanoparticles. As expected hybrid GNPs are better binders than the only sugar GNPs. The amino/ $\alpha$-Gal GNPs resulted to be the less efficient in inhibiting the migration of the pDNA under the condition used [Fig. 4(B)]. A light DNA fluorescence can still be observed even at high $\mathrm{w} / \mathrm{w}$ ratios, although basically all the plasmid is retained (Fig. 4(B), line 8). On the other hand, the amino/ $\beta$-Gal GNPs were able to retain the linear plasmid at w/w ratios between 50 and 100 (Fig. 4(D), lines 7-8). Of particular interest are the gels obtained with the mixed amino/ $\beta$-Glc GNPs [Fig. 4(F)]. Interaction between these GNPs and plasmids gave rise to bands with a drop-like shape, which might be caused by the formation of intermediate species as observed in AFM which may undergo conformational changes or degradation during migration. Still, at high concentration these GNPs were also able to inhibit migration of the pDNA (Fig. 4(F), line 8).

In conclusion, it was shown that AFM is a suitable technique to image the interaction between DNA and glyconanoparticles, especially using the method of immobilizing the DNA first, before allowing interaction with nanoparticles. In this way differences in binding could clearly be seen between the carbohydrate-bearing nanoparticles, and those modified with amino groups. It was also possible to distinguish different modes of binding when analyzing solutions containing DNA/nanoparticle mixtures, although in the case of the hybrid nanoparticles, there was some ambiguity in the interpretation of the AFM images alone, as the nature of the large globular feature was not clear. In this case, TEM images allowed the determination that the 
large features seen in the AFM were indeed large DNA/nanoparticle complexes, and the wide field imaging of the TEM showed that these complexes are remarkably uniform in morphology, and that all the nanoparticles were contained within these complexes. Imaging of these samples by AFM also indicated that all the DNA molecules were bound into these complexes. Finally, as shown in Fig. 2, in the case of the mixed amino/ $\alpha$-Gal nanoparticle and DNA solutions all the DNA was bound into large globular particles. In the case of the solutions of $\alpha$-Gal and $\beta$-Glc nanoparticles, AFM analysis revealed the presence of free DNA (and free nanoparticles) when the solution was dried onto mica. These were never seen in the case of the hybrid amino/ $\alpha$-Gal nanoparticles.

It was shown, therefore that hybrid amino/ $\alpha$-Gal nanoparticles make highly efficient, highly water soluble DNA-binders and condense the DNA into a compact globular shape, which is a desired property for gene-transfection agents [11]. Further work to prepare and characterize more effective amino/sugar GNPs for binding and transfecting DNA was already begun.

\section{ACKNOWLEDGMENT}

The authors would like to thank Dr. F. Pinto (IIQ-CSIC) for his discussions and help with gel electrophoresis experiments.

\section{REFERENCES}

[1] C. M. Niemeyer and M. Adler, "Nanoparticles, proteins, and nucleic acids: Biotechnology meets material science," Angew. Chem. Int. Ed., vol. 41, pp. 3779-3783, 2002.

[2] E. Katz and I. Willner, "Integrated nanoparticles-biomolecule hybrid systems: Synthesis, properties, and applications," Angew. Chem. Int. Ed., vol. 43, pp. 6042-6108, 2004.

[3] D. A. Muruve, "The innate immune response to adenivirus vectors," Hum. Gene Ther., vol. 15, pp. 1157-1166, Dec. 2004.

[4] K. Benihoud, P. Yeh, and M. Perricaudet, "Adenovirus vectors for gene delivery," Curr. Opin. Biotechnol., vol. 10, pp. 440-447, Oct. 1999.

[5] T. Montier, P. Delepine, C. Pichon, C. Ferec, D. J. Porteous, and P. Midoux, "Non-viral vectors in cystic fibrosis gene therapy: Progress and challenges," Trends Biotechnol., vol. 22, pp. 586-592, Nov. 2004.

[6] M. D. Brown, A. G. Schatzlein, and I. F. Uchegbu, "Gene delivery with synthetic (non-viral) carriers," Int. J. Pharm., vol. 229, pp. 1-21, Oct. 2001.

[7] K. Kostarelos and A. D. Miller, "Synthetic, self-assembly ABCD nanoparticles; a structural paradigm for viable synthetic non-viral vectors," Chem. Soc. Rev., vol. 34, pp. 970-994, Nov. 2005.

[8] J. Rojo, J. C. Morales, and S. Penadés, "Carbohydrate-carbohydrate interactions in biological and model systems," Top. Curr. Chem., vol. 218, pp. 45-92, 2002.

[9] M. A. Zanta, O. Boussif, A. Adib, and J. P. Behr, "In vitro gene delivery to hepatocytes with galactosylated polyethylenimine," Bioconjugate Chem., vol. 8, pp. 841-844, Nov.-Dec. 1997.

[10] J. Murata, Y. Ohya, and T. Ouchi, "Design of quaternary chitosan having antennary galactose residues as a gene delivery tool," Carbohyd. Polym., vol. 32, pp. 105-109, Feb. 1997.

[11] T. Ren, G. S. Zhang, and D. X. Liu, "Synthesis of galactosyl compounds for targeted gene delivery," Bioorg. Med. Chem., vol. 9, pp. 2969-2978, Nov. 2001.

[12] Y. Aoyama, "Macrocyclic glycoclusters: From amphiphiles trough nanoparticles to glycovirus," Chem.-Eur. J., vol. 10, pp. 588-593, Feb. 2004.

[13] D. Wakebayashi, N. Nishiyama, Y. Yamasaki, K. Itaka, N. Kanayama, A. Harada, Y. Nagasaki, and K. Kataoka, "Lactose-conjugated polyion complex micelles incorporating plasmid DNSA as a targetable gene vector system: Their preparation and gene transfecting efficiency against cultured HepG2 cells," J. Controlled Release, vol. 95, pp. 653-664, Mar. 2004.
[14] Y. Liu and T. M. Reineke, "Poly(glycoamidoamine)s for gene delivery. Structural effects on cellular internalization, buffering capacity, and gene expression," Bioconjugate Chem., vol. 18, pp. 19-30, 2006.

[15] Y. Liu and T. M. Reineke, "Hydroxyl stereochemistry and amine number within poly(glycoamidoamin)s affect intracellular DNA delivery," J. Amer. Chem. Soc., vol. 127, pp. 3004-3015, 2005.

[16] K. C. Wood, S. R. Little, R. Langer, and P. T. Hammond, "A family of hierarchically self-assembling linear-dendritic hybrid polymers for highly efficient targeted gene delivery," Angew. Chem. Int. Ed., vol. 44, pp. 6704-6708, 2005.

[17] C. M. McIntosh, E. A. Esposito, A. K. Boal, J. M. Simard, C. T. Martin, and V. M. Rotello, "Inhibition of DNA transcription using cationic mixed monolayer protected gold clusters," J. Amer. Chem. Soc., vol. 123, pp. 7626-7629, 2001.

[18] M. Thomas and A. M. Klibanov, "Conjugation to gold nanoparticles enhances polyethylenimine's transfer of plasmid DNA into mammalian cells," Proc. Nat. Acad. Sci., vol. 100, pp. 9138-9143, Aug. 2003.

[19] T. Kawano, M. Yamagata, H. Takahashi, Y. Niidome, S. Yamada, Y Katayama, and T. Niidome, "Stabilizing of plasmid DNA in vivo by PEG-modifies cationic gold nanoparticles and the gene expression assisted with electrical pulses," J. Controlled Release, vol. 111, pp. 382-389, Apr. 2006.

[20] G. Han, C. C. You, B. J. Kim, R. S. Turingan, N. S. Forbes, C. T. Martin, and V. M. Rotello, "Light-regulated release of DNA and its delivery to nuclei by means of photolabile gold nanoparticles," Angew. Chem. Int. Ed., vol. 45, pp. 3165-3169, 2006.

[21] J. M. De La Fuente, A. G. Barrientos, T. C. Rojas, J. Rojo, J. Canada, A. Fernandez, and S. Penades, "Gold glyconanoparticles as water-soluble polyvalent models to study carbohydrate interactions," Angew. Chem. Int. Ed., vol. 40, pp. 2258-2261, 2001.

[22] A. G. Barrientos, J. M. de la Fuente, T. C. Rojas, A. Fernandez, and S. Penades, "Gold glyconanoparticles: Synthetic polyvalent ligands mimicking glycocalix-like surfaces as tools for glycobiological studies," Chem.-Eur. J., vol. 9, pp. 1909-1921, May 2003.

[23] T. C. Rojas, J. M. De La Fuente, A. G. Barrientos, S. Penades, L. Ponsonnet, and A. Fernandez, "Gold glyconanoparticles as building blocks for nanomaterial design," Adv. Mater., vol. 14, pp. 585-588, Apr. 2002.

[24] J. M. de la Fuente, P. Eaton, A. G. Barrientos, M. Menendez, and S Penades, "Termodynamic evidence for $\mathrm{Ca} 2+$-mediated self-aggregation of Lewis X gold glyconanoparticles. A model for cell adhesion via carbohydrate-carbohydrate interaction," J. Amer. Chem. Soc., vol. 127, pp. 6192-6197, 2005.

[25] R. V. Duran, M. Hervas, B. De la Cerda, M. A. De la Rosa, and J. A. Navarro, "A laser flash-induced kinetic analysis of in vivo photosystem I reduction by site-directed mutants of plastocyanin and cytochrome $c_{6}$," Synechocystis, vol. 45, pp. 1054-1060, 2006, sp. PCC 6803 Biochemistry.

[26] T. Niidome, K. Nakashima, H. Takahashi, and Y. Niidome, "Preparation of primary amine-modified gold nanoparticles and their transfection ability into cultivated cells," Chem. Commun., 2004, 1978-1979.

[27] M. J. Hostetler, J. E. Wingate, C.-J. Zhong, J. E. Harris, R. W. Vachet M. R. Clark, J. D. Londono, S. J. Green, J. J. Stokes, G. D. Wignall, G. L. Glish, M. D. Porter, N. D. Evans, and R. W. Murray, "Alkanethiolate gold cluster molecules with core diameters from 1.5 to $5.2 \mathrm{~nm}$ : Core and monolayer properties as a function of core size," Langmuir, vol. 14, no. 1, pp. 17-30, 1998.

[28] A. Sanchez-Sevilla, J. Thimonier, M. Marilley, J. Rocca-Serra, and J. Barbet, "Accuracy of AFM measurments of the contour length of DNA fragments adsorbed on mica in air and in aqueos buffer," Ultramicroscopy, vol. 92, pp. 151-158, Aug. 2002.

[29] M. Ganguli, J. V. Babu, and S. Maiti, "Complex formation between cationically modified gold nanoparticles and DNA: An atomic force microscopic study," Langmuir, vol. 20, pp. 5165-5170, Jun. 2004.

[30] D. D. Dunlap, A. Maggi, M. R. Soria, and L. Monaco, "Nanoscopic structure of DNA condensed for gene delivery," Nucleic Acids Res., vol. 25, pp. 3095-3101, Aug. 1997.

[31] H. G. Abdelhady, S. Allen, M. C. Davies, C. J. Roberts, S. J. B. Tendler, and P. M. Williams, "Direct real-time molecular scale visualisation of the degradation of condensed DNA complexes exposed to DNase I," Nucleic Acids Res., vol. 31, pp. 4001-4005, Jul. 2003.

[32] Y. K. Jiao, D. I. Cherny, G. Heim, T. M. Jovin, and T. E. Schaffer, "Dynamic interactions of $\mathrm{p} 53$ with DNA in solution by time-lapse atomic force microscopy," J. Mol. Biol., vol. 314, pp. 233-243, Nov. 2001. 
Peter Eaton received the B.Sc. degree from the University of York, U.K., and the Ph.D. degree from Sheffield Hallam University, U.K., in 1998.

From 1998 to 2001 he was at the University of Portsmouth, U.K., using atomic force microscopy (AFM). From 2002 to 2004 he was with the IIQ, Seville, Spain, where he used AFM to study nanoparticle aggregation, and to study the interactions between nanoparticles and DNA. He is currently with REQUIMTE in Porto, Portugal, where he is working as an Associate Researcher and is using the AFM to study DNA-nanoparticle interactions and in a variety of other applications.

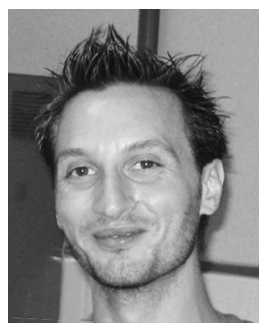

Andrea Ragusa received the degree in chemistry from the University of Parma, Italy, in 2001, under the supervision of Prof. Rocco Ungaro and Prof. Alessandro Casnati, and the Ph.D. degree from the University of Southampton, U.K., under the supervision of Prof. Jeremy D. Kilburn.

In 2001 he carried out a research project on the solubility in water of calixarenes. During the same period he spent a few months in Madrid, Spain, working for Prof. Javier de Mendoza. He then moved to England for his Ph.D. work. From March 2005 to December 2006 he waswith the IIQ, Seville, Spain, under the supervision of Prof. Soledad Penadés. During his postdoctoral stay he studied the interaction between metallic nanoparticles and DNA, with particular attention to their potential application in gene therapy. Since January 2007, he has been with the National Nanotechnology Laboratory, Lecce, Italy, where he is focusing on the biofunctionalization of metallic nanoparticles and nanocomposites for biomedical application. During his Ph.D. pursuit he studied the binding abilities of different types of carboxylate receptors, and in particular their enantioselective properties.
Caroline Clavel photograph and biography not available at the time of publication.

Cristina T. Rojas photograph and biography not available at the time of publication.

Paul Graham photograph and biography not available at the time of publication.

Raúl V. Durán photograph and biography not available at the time of publication.

Soledad Penadés photograph and biography not available at the time of publication. 